\title{
Modeling the integration of bacterial rRNA fragments into the human cancer genome
}

Karsten B. Sieber ${ }^{1}$, Pawel Gajer ${ }^{1,2}$ and Julie C. Dunning Hotopp ${ }^{1,2,3^{*}}$

\begin{abstract}
Background: Cancer is a disease driven by the accumulation of genomic alterations, including the integration of exogenous DNA into the human somatic genome. We previously identified in silico evidence of DNA fragments from a Pseudomonas-like bacteria integrating into the 5'-UTR of four proto-oncogenes in stomach cancer sequencing data. The functional and biological consequences of these bacterial DNA integrations remain unknown.

Results: Modeling of these integrations suggests that the previously identified sequences cover most of the sequence flanking the junction between the bacterial and human DNA. Further examination of these reads reveals that these integrations are rich in guanine nucleotides and the integrated bacterial DNA may have complex transcript secondary structures.
\end{abstract}

Conclusions: The models presented here lay the foundation for future experiments to test if bacterial DNA integrations alter the transcription of the human genes.

Keywords: DNA integration, Somatic genome, Genome variation, Cancer genomics, Host-bacteria interactions

\section{Background}

The advent of next generation sequencing has enabled the interrogation of the human genome and transcriptome with base pair resolution. This new window into the human genome has expanded our understanding of important somatic genome variants such as single nucleotide polymorphisms [1-4], chromosomal rearrangements [5-7], and exogenous DNA integrations [8, 9]. These variants have primarily been identified using paired-end sequencing, a process that relies on breaking the genomic DNA into many small fragments and sequencing each fragment of DNA from the ends inward toward the center. The resulting pairs of sequencing reads share a unique relationship as they originated from the same randomly sheared DNA fragment.

By leveraging the unique relationship between the paired-end reads, chromosomal rearrangements and integrations of exogenous DNA can be identified and characterized [6]. For example, a rearrangement between

\footnotetext{
* Correspondence: jdhotopp@som.umaryland.edu

${ }^{1}$ Institute for Genome Science, University of Maryland School of Medicine,

Baltimore, MD 21201, USA

${ }^{2}$ Department of Microbiology and Immunology, University of Maryland

School of Medicine, Baltimore, MD 21201, USA

Full list of author information is available at the end of the article
}

two chromosomes would result in at least one chimeric chromosome (Fig. 1). By mapping the sequencing reads to a reference genome, a subset of the paired-end reads will support the chromosomal rearrangement by spanning the break point between the two chromosomes with one of the paired-end reads mapping to chromosome $\mathrm{A}$, while the respective other paired-end read maps to chromosome B (Fig. 1). In addition to identifying rearrangements, this technique has also been applied to identify integrations into the human genome of: transposable elements [10-12], viral genomes [8,9], and bacterial DNA [13]. If the integration site can be rebuilt in silico, the structure and sequence of the integration site can be used to determine a potential mechanism of integration [14, 15], elucidate potential functional implications of novel integrations, and lay the foundation for future experiments.

Previously, we identified paired-end reads supporting the integration of Pseudomonas-like rRNA genes into the 5'-UTR of genes in human stomach adenocarcinoma (STAD) genomes using RNA-Seq data from The Cancer Genome Atlas (TCGA) [13]. These paired-end reads have one read mapping exclusively to the $16 \mathrm{~S}$ or $23 \mathrm{~S}$ rRNA genes of a Pseudomonas-like bacteria [13], while the paired read maps uniquely to the $5^{\prime}$-UTR of the 


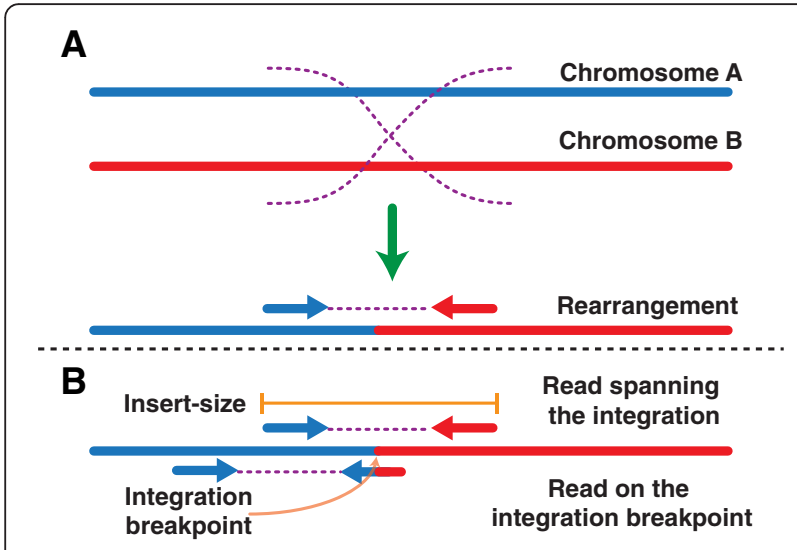

Fig. 1 A brief schematic illustrates using next generation sequencing to identify structural variation. A recombination event is illustrated between two chromosomes (a). In order to identify structural variation, paired-end reads are identified that map to different chromosomes indicating a fusion of the two different chromosomes has occurred (b)

human CEACAM5, CEACAM6, CD74, or TMSB10 genes. As such, these read pairs support bacterial DNA integrations by spanning the junction of the bacterial and human DNA. To identify these regions in the human genome, $>4 \times$ human coverage in a single sequencing run was required. Despite this level of coverage, single reads that traverse the integration site were not identified, likely owing to the length of the sequencing reads and limits of alignment algorithms. Such reads would enable the assembly of the integration with the base pair resolution needed to determine the potential mechanism of integration [5]. Instead, here, we have used the paired-end reads to model the most likely structure of the integration of Pseudomonas-like rRNA gene fragments into CEACAM5, CEACAM6, CD74, and TMSB10.

\section{Results \\ Establishing the boundaries of the bacterial rRNA integrations}

In all cases, reads were only recovered spanning one side of the junction between bacterial and human DNA, likely because the integration is close to the transcriptional start site. Despite numerous attempts, the integrations could not be assembled using the original reads supporting an integration event or with additional reads located near the integrations that were identified using either alignment- or BLASTbased algorithms. Neither split reads, those that are part bacterial and part human on the breakpoint of the integrations, nor soft-clipped reads could be identified in the original alignments or in alignments to a custom reference with both the human genome and the Pseudomonas $16 \mathrm{~S} \& 23 \mathrm{~S}$ rRNA genes. Further BLAST-based examination of the unmapped read in read pairs that had only one read aligned to the human genome in the region flanking the integration or the Pseudomonas rRNA gene reference near the integration also failed to identify split reads.

To examine this further, a bacterial-human DNA integration was constructed with the bacterial DNA directly abutting the human DNA. A mock dataset was created of all 101 possible combinations of 100 -bp paired end reads spanning the integration breakpoint in this artificial sequence mimicking a bacterial-human DNA integration. The first read generated was entirely bacterial and ended at the integration breakpoint. Each of the 100 subsequent reads in the mock dataset shifted by 1-bp, such that the dataset included a mock read for every position across the integration beginning with an entirely bacterial read and ending with an entirely human read. The second read in the pair was held constant and corresponded to a sequence 225-bp downstream of the break point. LGTSeek identified only 3 (3\%) reads that cover the breakpoint, none of which were soft-clipped as the differences with respect to mapping were similar to those arising from sequencing errors. Therefore, we conclude that LGTSeek, and more specifically the version of BWA used in LGTSeek, is unable to identify reads that span the junction between bacterial and human DNA in this data set.

Given that the bacterial DNA integrations could not be assembled, the focus shifted to estimating the location of the bacterial rRNA gene fragment integrations into the human genome by examining the structure of the human transcript and the reads supporting the bacterial DNA integrations. The integration breakpoint must be downstream of the transcriptional start site (TSS) of each human gene for three reasons. First, the integrations were identified in an RNA-Seq data set derived from transcripts so they must be within the transcript boundaries. Second, examination of the expression of these genes across all participants from the STAD and Breast Cancer (BRCA) data sets from TCGA data available for download from the SRA between September $18^{\text {th }}-20^{\text {th }}, 2011$ [13] are consistent with an accurately annotated TSS (Additional file 1: Figure S1). Third, the introns and other noncoding regions of the human genome do not have low-level sequencing coverage that would suggest the presence of contaminating genomic DNA. Therefore, the integration must be downstream of the annotated TSS and the TSS is the left-most boundary for the possible location of bacterial DNA integration, relative to the direction of transcription.

The right-most boundary for the bacterial DNA integration break point can be delineated from the position of human reads supporting the bacterial DNA integrations. More specifically, the bacterial DNA integration must be upstream of the human reads supporting the bacterial 
DNA integration, relative to the direction of transcription. Therefore, the left most boundary for the site of integration in the human genes, in all cases, should be considered the TSS, while the right most boundary position for the integration is the left most position of the consensus sequence for the human reads supporting the bacterial DNA integration.

\section{Using the library insert-size to refine the location of bacterial DNA integration}

The library insert-size and its distribution can be used to refine the location of the bacterial rRNA gene fragment integration. In order to model the integrations with the greatest resolution and accuracy, two calculations were used to determine the number of bases needed between the bacterial and human consensus sequences to mirror the library insert-size distribution.

Suppose that the distance between the bacterial and human fragments is $x$, where $0 \leq x \leq 100$ (Fig. 2a), and that each integration has $n$ total reads supporting it. The insert-size, $I_{i}(x)$, of the $i^{\text {th }}$ read pair spanning the junction is calculated based on the known positions of each read within the bacterial and human fragment consensus sequences and the assumed distance, $x$, between these fragments. The mean absolute value, $A D(x)$, of the differences between the median sequencing library insertsize, $M_{S L}$, and the read pairs supporting the integration can be calculated as $A D(x)=\frac{\sum_{i=1}^{n}\left|I_{i}(x)-M_{S L}\right|}{n}$, where $n$ is the total number of reads supporting the integration. The location of the junction between bacterial rRNA and the human gene of interest was estimated to be within the range, $\left[L-x_{A D}, L\right]$, where $L$ is the position of the left end of the human fragment consensus sequence within the human gene and $x_{A D}$ is the value of $x$ which has the minimum value of $A D(x)$ (Fig. 2b).

The second approach of estimating the distance between the bacterial and human consensus sequences uses a dissimilarity measure called the Jensen-Shannon distance, $J S D(x)$, between the insert-size distributions of
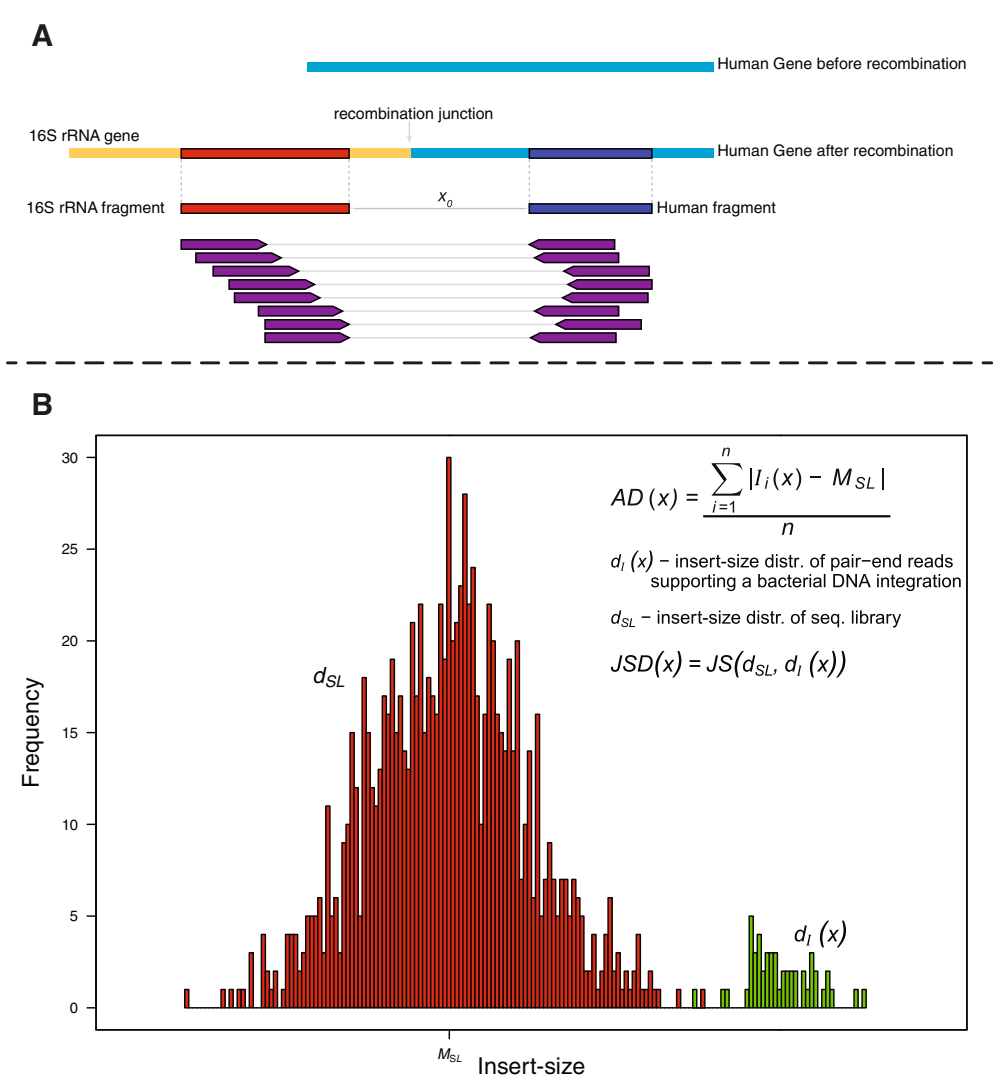

Fig. 2 The location of the bacterial DNA integration was refined using the library insert-size. In this hypothetical case, the paired-end reads support the integration of a fragment of the bacterial $16 \mathrm{~S}$ rRNA gene into a human gene (a). Using the consensus sequence of the two fragments, the number of bases, $x x$, needed between the two fragments was titrated so that the insert-sizes of the reads supporting the integration most closely resemble those of the sequencing library. Two calculations were used to determine the optimum distance between the two fragments. The first calculation identified the number of bases $(x)$ yielding the minimum average difference (AD) between insert-size of the reads supporting the integration, $l_{i}(x)$, and the median sequencing library insert-size, $M_{\boldsymbol{S L}}$. The second calculation determined the number of bases $(\boldsymbol{x})$ resulting in the minimum Jensen-Shannon distance (JSD) for the insert-size distributions of the sequencing library, $d_{\mathbf{S L}}$ and the reads supporting the integration, $d_{\boldsymbol{I}}(x)(\mathbf{b})$ 
the sequencing library, $d_{S L}$, and reads supporting the integration, $d_{I}(x)$ (Fig. 2b). The Jensen-Shannon distance between two distributions takes values between 0 and 1 , with 0 implying the identity of the distributions and 1 indicating major discordance between the distributions. As in the average distance case, the location of the junction between bacterial $16 \mathrm{~S}$ rRNA fragment and the human gene of interest was estimated to lie within the range, $\left[L-x_{J S}, L\right]$, where $L$ is the position of the left end of the human fragment consensus sequence within the human gene and $x_{J S}$ is the distance value for the minimum value of $J S D(x)$ (see methods for further details). The majority of the time the AD and JSD calculations were in agreement. When the two calculations differed, the JSD was preferred since it accounts for the distribution of insert size values for the two populations.

\section{Validating the method}

To validate this method of modeling the bacterial DNA integrations, a bacterial DNA integration in a cancer cell line genome was identified, modeled, and experimentally validated. Using the Cancer Cell Line Encyclopedia [16] and a similar cancer cell line sequencing project that is publicly available through Genentech Inc [17], pairedend RNA-Seq reads were identified that support the likely in vitro integration of a kanamycin resistance gene (aminoglycoside-3'-O-phosphotransferase) near the 67,142 kbp position in chromosome 6 of the KPL-1 cell line. The paired-end reads supporting this integration spanned the junction of bacterial and human DNA at the $3^{\prime}$ - side of the kanamycin gene, with respect to the direction of transcription. Given that this integration was likely in vitro, a viral promoter is expected to drive the kanamycin gene expression, which would prevent the bacterial sequence focused pipeline from detecting the $5^{\prime}$ - junction between the integrated bacterial DNA and human chromosome. In both independently sequenced datasets, the JSD and the $\mathrm{AD}$ predicted that there should been 0 bp between the consensus bacterial and human DNA (Fig. 3a, b). Subsequently, the genomic DNA of the KPL-1 cell line was acquired from Leibniz Institute DSMZ and the junction between the integrated kanamycin resistance gene and chromosome 6 was PCR amplified, cloned, and sequenced. The sequencing revealed that the JSD method correctly predicted the sequence and approximate position of the integration site, but that the exact position was off by $2 \mathrm{bp}$, with the actual position of integration being $2 \mathrm{bp}$ from that predicted by the model (Fig. 3c).

\section{KPL1 breakpoint}

While investigating the KPL1 integration, soft-clipped reads at the KPL1 bacteria-human DNA junction were identified manually. To better understand why these reads were not identified by the LGTSeek pipeline, a

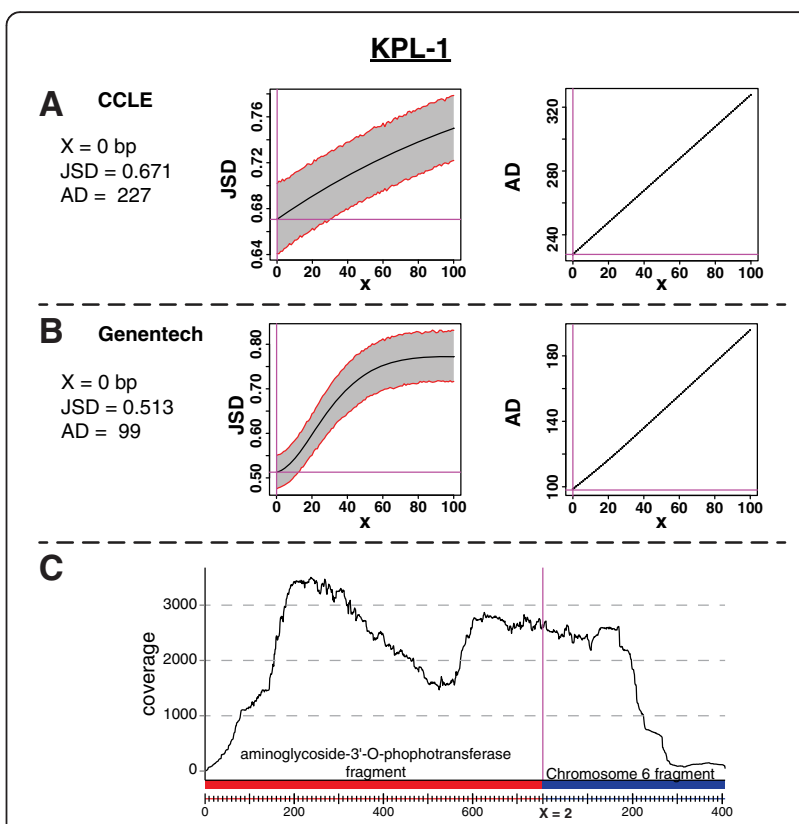

Fig. 3 A bacterial DNA integration into the KPL-1 cell line genome was modeled using the JSD and AD calculations. Based on the data from the Cancer Cell Line Encyclopedia [16] and a similar data set from Genentech [17], the JSD and AD methods predicted that the bacterial \& human consensus sequences are $0 \mathrm{bp}$ apart $(\mathbf{a}, \mathbf{b})$. However, PCR amplification and sequencing revealed an additional $2 \mathrm{bp}$ of sequence between the two consensus sequences as illustrated (c). Consistent with this, a plot of the sequence coverage of the reads across the junction is relatively constant (c). No further insertions or deletions are observed in the underlying reads (data not shown)

mock dataset was created of all 101 possible combinations of 100-bp paired end reads spanning the cloned and sequenced integration breakpoint. The first read generated was entirely bacterial and ended at the integration breakpoint. Each of the 100 subsequent reads in the mock dataset shifted by 1-bp, such that the dataset included a mock read for every position across the integration beginning with an entirely bacterial read and ending with an entirely human read. The second read in the pair was held constant and corresponded to a sequence 190-bp downstream of the break point, which was selected based on the insert-size distribution of the CCLE data. No split reads were identified from running the entire KPL1 dataset spiked with this mock dataset through the LGTSeek pipeline due to a 24-bp inversion in the bacterial DNA near the KPL1 breakpoint (Additional file 2: Figure S2).

\section{Bacterial rRNA gene integrations into CEACAM5 \& CEACAM6}

From the TCGA STAD data set that was previously analyzed [13], two participants have paired-end reads supporting Pseudomonas DNA integrating into exon 1 of CEACAM5 [GenBank:NM_004363.3]. In participant A, 
the integration of the Pseudomonas 16S rRNA gene [GenBank:M34133.1] fragment is supported by 8 pairedend reads (Fig. 4a). Using the JSD, the calculated distance between the bacterial and human fragment consensus sequences is $26 \mathrm{bp}$, with a minimum JSD value of 0.748 (Fig. 4b), while the AD placed it between 11-17 bp (Fig. 4c). A visual inspection of the read insert-size distribution over a variety of values of $x$ (Fig. 4d-h) further supports the JSD prediction. Therefore, given that the human fragment consensus sequence starts at position 74 in the CEACAM5 gene, the bacterial DNA integration is estimated to occur between positions 48-73 in the CEACAM5 gene (Fig. 4f).

Participant $\mathrm{B}$ has 11 paired-end reads supporting the Pseudomonas 16S rRNA gene integration into CEACAM5 (Fig. 5a). Both the JSD and the AD calculations are in agreement that the distance between the two fragments is $0 \mathrm{bp}$ (Fig. 5b, c), and that the 16S rRNA gene fragment has integrated at the $18 \mathrm{bp}$ in CEACAM5. However, manually inspecting the insert-size of the reads suggests that the human read farthest upstream is an outlier that does not fit the insert-size distribution of the other reads (Fig. 5a). After removing this potential outlier, a model of the integration was calculated (Fig. 5d). The JSD and AD support that the distance between the consensus sequences is 7 bp (Fig. 5e, f). In addition, both the JSD and AD decreased (Table 1), suggesting that this may be a more accurate representation of the integration, particularly given that decreasing the number of reads in the analysis typically increases the JSD as described below. Therefore, the integration of the $16 \mathrm{~S}$ rRNA gene fragment is likely between positions 4753 bp of CEACAM5. For the subsequent comparisons in this manuscript, only this latter model is discussed.

If the potential outlier paired-end read is excluded, the modeling suggests that the $16 \mathrm{~S}$ rRNA integrations into the CEACAM5 gene of participants A \& $\mathrm{B}$ are nearly identical (Table 1 ), with the models only differing by a few bp (354-454 \& 331-449 bp). In addition, the human reads from participants A \& B map to similar positions in CEACAM5 (74-147 \& 54-148 bp, respectively).

In addition to the 16S rRNA gene integration described above, participant $\mathrm{A}$ also has 3 paired-end reads supporting an integration of 1626-1699 bp from the Pseudomonas 23S rRNA gene [GenBank:Y00432.1] into the CEACAM5 gene. The JSD and AD calculations support that the integration is between positions 49-80 of CEACAM5. In addition, participant $\mathrm{B}$ has 2 reads supporting integration of a nearly identical fragment of the $23 \mathrm{~S}$ rRNA gene (1626-1701 bp) integrating into an almost identical region in CEACAM5 (positions 59-93). Therefore, the data support two different Pseudomonas DNA integrations into approximately the same position in $C E A$ CAM5 for both participants A \& B.
Similar to CEACAM5, participants A \& C have similar Pseudomonas 16S rRNA gene fragment integrations into the related CEACAM6 [GenBank:NM_002483.6]. Both participants have nearly identical fragments of the $16 \mathrm{~S}$ rRNA gene (329-454 \& 321-456 bp) integrated into CEACAM6 at the same predicted position (59-77 \& 50$72 \mathrm{bp}$ ) (Table 1). In addition, these CEACAM6 integrations are similar to the (a) position of the $16 \mathrm{~S} \& 23 \mathrm{~S}$ rRNA integrations and (b) the sequence of the $16 \mathrm{~S}$ rRNA integrations into CEACAM5.

\section{CD74 integrations}

Both participants D and E have fragments of the Pseudomonas $16 \mathrm{~S}$ and 23S rRNA integrated in the CD74 gene [GenBank:NM_001025159.2]. Both participants have almost identical fragments of the 16S rRNA gene (563708 \& 589-697 bp) integrated into overlapping positions in the CD74 gene. For participant D, the calculated model supports that the $16 \mathrm{~S}$ rRNA fragment integrated between positions 180-187 bp of CD74, while the fragment of $16 \mathrm{~S}$ rRNA integrated into participant E's CD74 gene between positions 163-205 bp (Table 1). The data supporting these two integrations are consistent and suggests that the two participants have similar integrations. The data also support a second fragment further downstream in the 16S rRNA gene (927-1022 bp) integrating into positions 136-147 bp of CD74 in participant D.

There are 3 different fragments of the 23S rRNA gene fragments with support for integration into the $C D 74$ gene of participants D \& E. All three integrations have similar locations in the CD74 gene ( 190-290 bp) (Table 1). Of the three integrations, two integrations, one each from participant $\mathrm{D} \& \mathrm{E}$, are predicted to have integrated into the same region of CD74 (167-195 \& 170-191 bp). The remaining 23S rRNA gene fragment with support for integration into $C D 74$ is predicted to have integrated into the 201-225 bp of CD74 of participant E (Table 1).

\section{TMSB10 integrations}

Unlike the bacterial DNA integrations into CEACAM5, $C E A C A M 6$, and CD74, the TMSB10 gene [GenBank: NM_021103.3] has only one participant with the integration of a Pseudomonas 16S rRNA fragment (701-927 bp). The model estimates that the 16S rRNA fragment integrated into the first $13 \mathrm{bp}$ of TMSB10 (Table 1).

\section{Calibrating the JSD}

As described previously, a calculated JSD value of 0 supports that the two populations are identical, while a value of 1 indicates major discordance between the distributions. The average JSD for the models of bacterial DNA integration presented here was 0.78 . Therefore, the JSD calculation was investigated further to determine why it was not closer to zero. To test the effect that the 


\section{A}

\section{Participant A}

\section{Pseudomonas 16S \& CEACAM5}
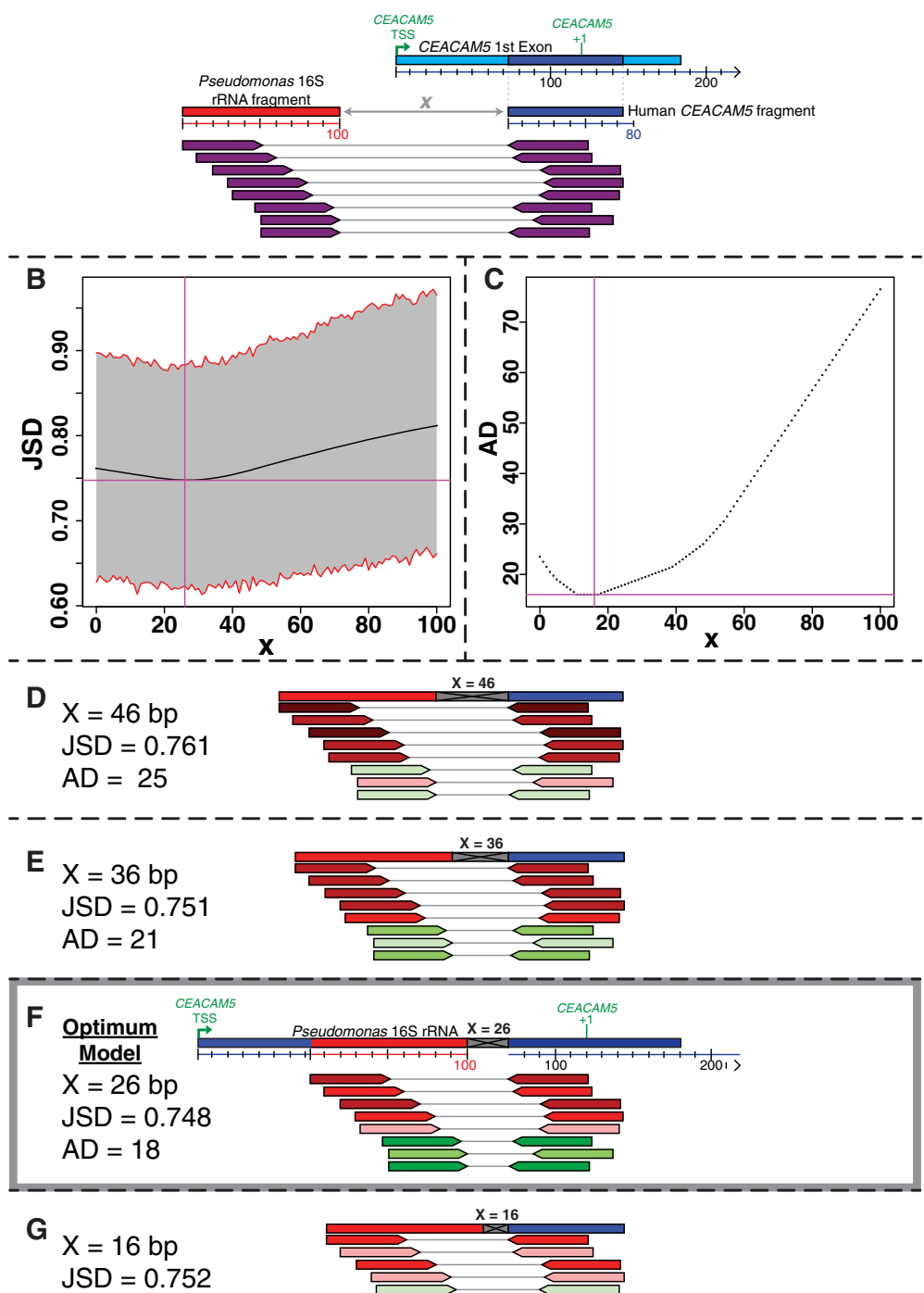

$\mathrm{JSD}=0.752$

$\mathrm{AD}=16$

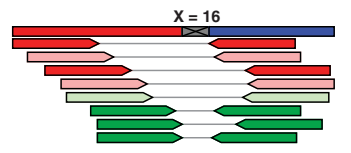

$\mathrm{H} \mathbf{X}=6 \mathrm{bp}$

$\mathrm{JSD}=0.758$

$\mathrm{AD}=19$

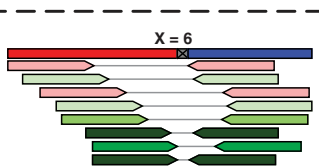

Fig. 4 The model of the Pseudomonas 16S rRNA gene fragment integration into CEACAM5 of participant A is presented. The structure of the Pseudomonas 165 rRNA gene fragment, the first exon CEACAM5, and the paired reads supporting the integration of the rRNA gene fragment into CEACAM5 are illustrated (a). Calculations using the JSD (b) and AD (c) support that there are 26 bp between the Pseudomonas 165 rRNA \& CEACAM5 fragments and that the integration is in 48-73 bp of CEACAM5 ( $\mathbf{f}$. The CEACAM5 sequence upstream of the bacterial 16S rRNA fragment is for illustrative purposes only ( $\mathbf{f}$ ). The insert-size for each paired-end read is color-coded with lighter colors being closer to the median insert-size, red designating those insert-sizes larger than the median, and green for insert-sizes less than the median as further clarified in Additional file 7: Figure S7. The optimum model for the structure of the integration $(\mathbf{f})$ is compared to alternative distances $(x)$ between the two fragments in (d-h), where an additional 1.0, 0.5, $-0.5, \&-1.0$ median absolute deviations ( $20 \mathrm{bp}$ ) are placed between the two fragments represented by the gray region. The actual DNA sequence of the gray region is unknown

number of reads had on the JSD, the JSD was calculated for 1000 samples for specific values of $k$, such that $0 \leq k$ $\leq 100,000$, where $k$ represents the number of randomly sampled read pairs that mapped to the CEACAM5 transcript from the sequencing library of participant $B$ (Fig. 6). Samples with 3-10 randomly selected reads had 
A
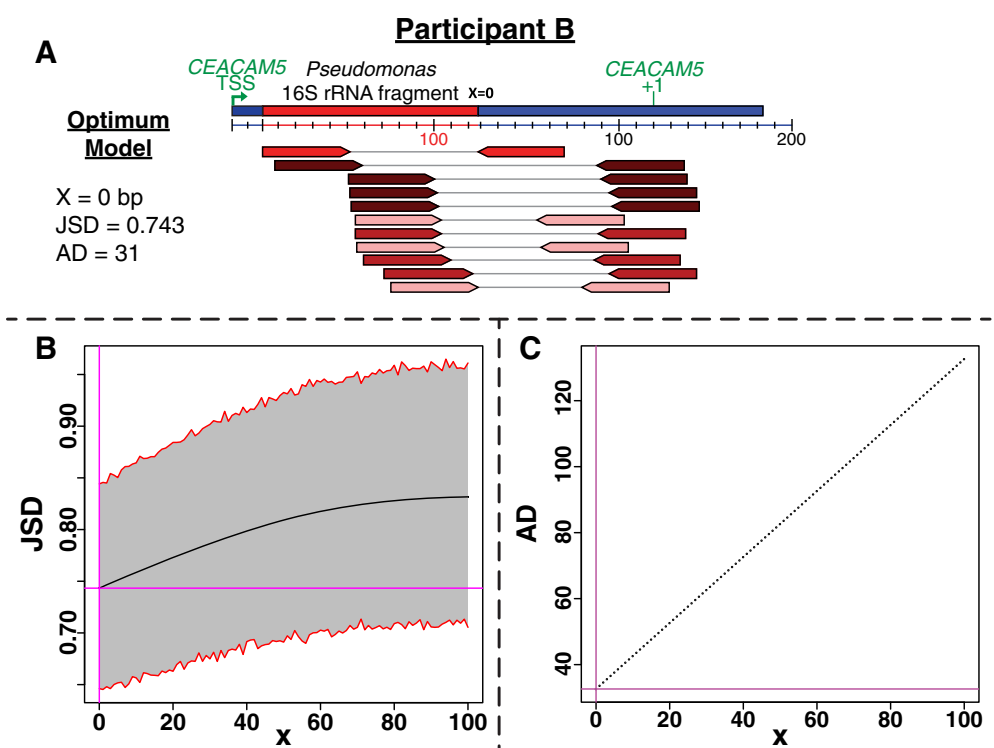

D

Participant B Adjusted

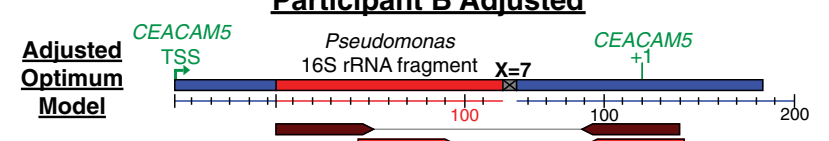

$\mathrm{X}=7 \mathrm{bp}$

$\mathrm{JSD}=0.722$

$A D=19$
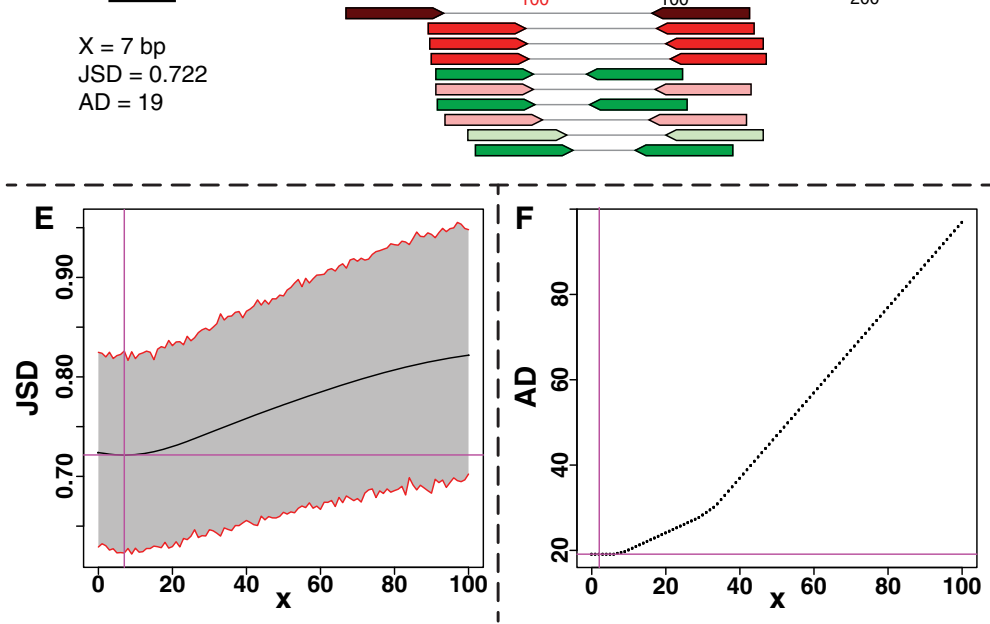

Fig. 5 A model of the Pseudomonas 165 rRNA gene fragment integration at the $18 \mathrm{bp}$ of CEACAM5 from participant B is illustrated (a). Based on the JSD (b) and AD (c) calculations, the model has 0 bp between the bacterial and human fragment consensus sequences. After removing the potential outlier paired-end read, the optimum structure for the integration is illustrated in $\mathbf{d}$. By removing the potential outlier read, both the JSD and AD decreased $(\mathbf{e} \& \mathbf{f})$, supporting that there are 7 bp between the two fragments. This indicates the Pseudomonas 165 rRNA likely integrated between positions 45-53 bp of CEACAM5

a JSD of $\sim 0.78$, consistent with the results for modeling the integrations. As the number of randomly sampled reads increases, the JSD decreases. The average JSD continues to decrease as the number of reads increases until around 5000 reads when the JSD starts to plateau, eventually reaching a minimum of 0.07 at 100,000 reads, the maximum number tested.

\section{Guanine rich sequences}

While modeling the integrations, it was observed that both the bacterial and human fragment consensus sequences seemed to be particularly rich in guanine. Consistent with this observation, the median \%GC for the bacterial and human fragment consensus sequences was $52.7 \%$ and $58.5 \%$, respectively. The \%GC and \%AG were compared for the consensus sequences supporting the bacterial DNA integrations using the Wilcoxon rank-sum test (WRT) (Table 2, Additional file 3: Figure S3). The bacterial and human consensus sequences were significantly different than each other (Table 2). Both sets of consensus sequences were also significantly different than the complete RefSeq Pseudomonas genomes 
Table 1 Summary of the bacterial DNA integration models

\begin{tabular}{|c|c|c|c|c|c|c|c|}
\hline Name & Min JSD & JSD dist. bp & Integration site & Min. AD & $A D$ dist. bp & Bacterial fragment & Human fragment \\
\hline A_16S_C5 & 0.748 & 26 & $48-73$ & 16 & $11-17$ & $354-454$ & $74-147$ \\
\hline A_23S1_C5 & 0.776 & 22 & $51-72$ & 4.33 & 33 & 1308-1385 & $73-145$ \\
\hline A_23S2_C5 & 0.785 & 32 & $49-80$ & 12.67 & 29 & $1626-1699$ & $81-157$ \\
\hline B_16S_C5 & 0.743 & 0 & 18 & 30.82 & 0 & $324-449$ & $19-148$ \\
\hline B_16Sa_C5 & 0.722 & 7 & $47-53$ & 19.10 & $0-6$ & $331-449$ & $54-148$ \\
\hline B_23S_C5 & 0.795 & 35 & $59-93$ & 12 & $30-54$ & $1626-1701$ & $94-145$ \\
\hline A_16S_C6 & 0.768 & 19 & $59-77$ & 17.60 & 17 & $329-454$ & $78-168$ \\
\hline C_16S_C6 & 0.720 & 23 & $50-72$ & 17.89 & 31 & $321-456$ & $73-140$ \\
\hline D_16S1_CD & 0.802 & 2 & 180-187 & 27.50 & $0-20$ & $563-708$ & $188-278$ \\
\hline D_16S2_CD & 0.802 & 41 & $136-147$ & 26.50 & $7-60$ & $927-1022$ & $184-241$ \\
\hline D_23S_CD & 0.815 & 22 & 167-195 & 166.75 & 0 & 892-1323 & 196-306 \\
\hline E_16S1_CD & 0.809 & 48 & $163-205$ & 46 & $0-65$ & 589-697 & 206-290 \\
\hline E_23S1_CD & 0.791 & 31 & $201-225$ & 0.50 & $44-45$ & $907-978$ & $226-296$ \\
\hline E_23S2_CD & 0.782 & 28 & 170-191 & 29 & $0-38$ & 1255-1379 & $192-269$ \\
\hline F_16S_T10 & 0.812 & 19 & $(-5)-13$ & 169.44 & 0 & $701-927$ & 14-196 \\
\hline
\end{tabular}

Names indicate the participant with letters $A$ through $F$, their respective rRNA gene integration $(16 \mathrm{~S}, 23 \mathrm{~S})$, and the human gene it has integrated into $(C 5=C E A C A M 5$, $\mathrm{C} 6=$ CEACAM6, $\mathrm{CD}=\mathrm{CD74}, \mathrm{T} 10=\mathrm{TMSB10}$ )

and the human genomic reference (Table 2). The human fragment consensus sequences were also significantly different that the Pseudomonas 16S \& 23S rRNA genes available in the Silva database (Table 2). Considering that the predicted integration sites are typically into the first $150 \mathrm{bp}$ of each human transcript, the \%GC of the fragment consensus sequences supporting the integrations was compared with the first $150 \mathrm{bp}$ of each transcript from the human transcriptome reference and found that the \%GC for the human fragment consensus sequences was significantly different (Table 2). Given that the expressed transcripts may have a different GC profile [18], the \%GC for the fragment consensus sequences was compared to the expressed transcripts ( $>0$ RPKM) from the participants with the bacterial DNA integrations. The \%GC of the human consensus sequences was significantly different than the expressed transcripts (Table 2). Next, by stratifying the expressed genes into quartiles based on their RPKM, the \%GC for the quartiles of expressed transcripts was compared to consensus sequences. The human fragment consensus sequences were significantly different than the two most highly
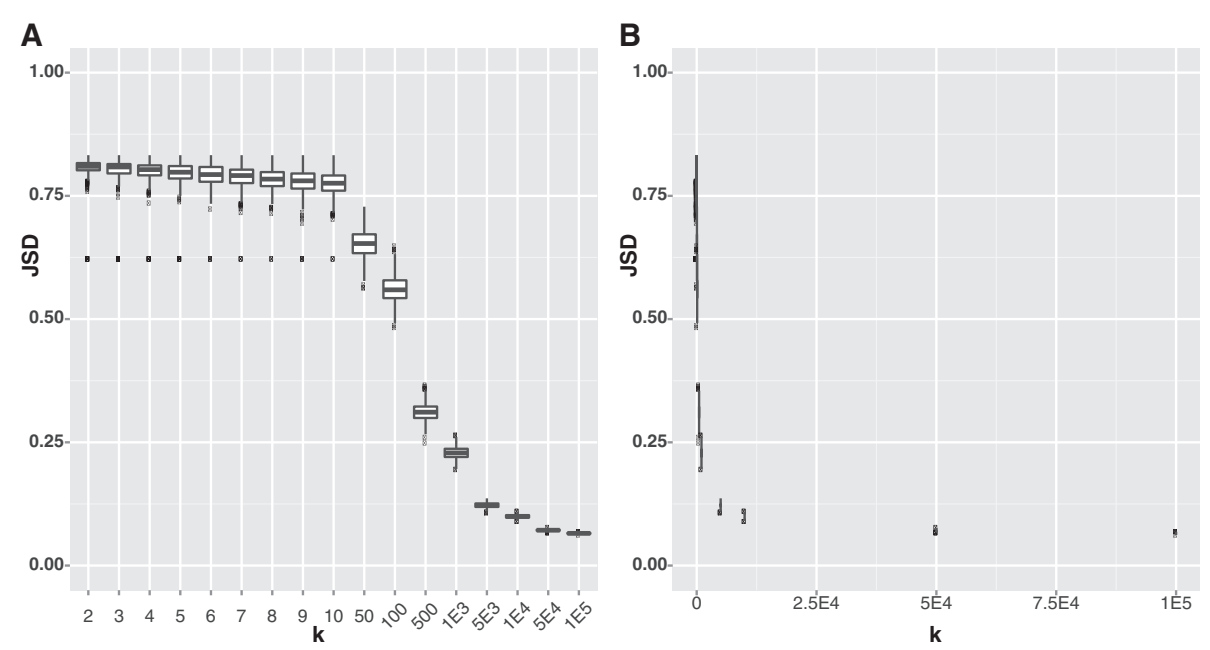

Fig. 6 Boxplots illustrate the JSD (y-axis) calculated for subsets of RNA-Seq data mapped to the human transcript. The insert-size distribution of the sequencing library of participant B was compared to the distribution of insert-sizes of specific values of randomly selected reads that mapped to the CEACAM5 transcript from the sequencing library of participant $B$ (a). Panel A has a discontinuous $x$-axis to better illustrate the JSD over the various number of reads tested $(\mathrm{k})$; panel $\mathbf{b}$ illustrates the same data with a continuous $x$-axis 
Table 2 Wilcoxon rank-sum test comparison of GC \& AG content

\begin{tabular}{|c|c|c|c|c|}
\hline \multirow[t]{2}{*}{ References } & $\begin{array}{l}\text { \%GC of human } \\
\text { fragment consensus } \\
\text { sequences }\end{array}$ & $\begin{array}{l}\% G C \text { of bacterial } \\
\text { fragment consensus } \\
\text { sequences }\end{array}$ & $\begin{array}{l}\text { \%AG of human } \\
\text { fragment consensus } \\
\text { sequences }\end{array}$ & $\begin{array}{l}\% A G \text { of bacterial } \\
\text { fragment consensus } \\
\text { sequences }\end{array}$ \\
\hline & $p$-value & $p$-value & $p$-value & $p$-value \\
\hline Human fragment consensus sequences & NA & $9.5 \mathrm{e}-04^{*}$ & NA & $1.5 \mathrm{e}-01$ \\
\hline Bacterial fragment consensus sequences & $9.5 e-04^{*}$ & NA & 0.15 & NA \\
\hline Pseudomonas $16 \mathrm{~S}$ rRNA genes & $8.3 e-06^{*}$ & 7.7e-02 & 1.0 & $8.4 \mathrm{e}-08^{*}$ \\
\hline Pseudomonas $23 \mathrm{~S}$ rRNA genes & $2.2 \mathrm{e}-10^{*}$ & 1.0 & 1.0 & $5.3 e-09^{*}$ \\
\hline Pseudomonas genomes & $6.4 \mathrm{e}-05^{*}$ & $7.2 \mathrm{e}-08^{*}$ & NA & NA \\
\hline Human genome & $6.8 \mathrm{e}-09^{*}$ & $1.2 \mathrm{e}-06^{*}$ & NA & NA \\
\hline Human transcriptome & $8.0 \mathrm{e}-06^{*}$ & $1.8 \mathrm{e}-02$ & 1.0 & $7.3 e-02$ \\
\hline Human transcriptome first $150 \mathrm{bp}$ & $5.7 e-05^{*}$ & $5.2 \mathrm{e}-02$ & 1.0 & 1.0 \\
\hline Human genes with integrations & $8.1 \mathrm{e}-01$ & 1.0 & 0.64 & $7.8 \mathrm{e}-02$ \\
\hline Human genes with integrations first 150 bp & 1.0 & $6.3 e-02$ & 1.0 & $2.6 \mathrm{e}-01$ \\
\hline Expressed human transcripts & $2.9 \mathrm{e}-03^{*}$ & 1.0 & $1.7 \mathrm{e}-3^{*}$ & $2.0 \mathrm{e}-09^{*}$ \\
\hline Q1 Expressed human transcripts & $1.2 \mathrm{e}-02$ & $3.5 \mathrm{e}-01$ & 7.3-e $4^{*}$ & $1.4 \mathrm{e}-09^{*}$ \\
\hline Q2 Expressed human transcripts & $8.0 \mathrm{e}-03$ & $6.4 \mathrm{e}-01$ & $1.1 e-3^{*}$ & $1.7 e-09^{*}$ \\
\hline Q3 Expressed human transcripts & $2.1 \mathrm{e}-03^{*}$ & $6.1 \mathrm{e}-01$ & $2.5 \mathrm{e}-3^{*}$ & $2.2 \mathrm{e}-09^{*}$ \\
\hline Q4 Expressed human transcripts & $2.7 e-04^{*}$ & $2.4 \mathrm{e}-01$ & $4.4 \mathrm{e}-4^{*}$ & $3.4 \mathrm{e}-09^{*}$ \\
\hline Expressed human transcripts first $150 \mathrm{bp}$ & 1.0 & 4.3e-01 & 3.7e-2 & $4.6 \mathrm{e}-07^{*}$ \\
\hline Q1 Expressed human transcripts first $150 \mathrm{bp}$ & 1.0 & $3.5 \mathrm{e}-01$ & $1.7 e-2$ & $1.3 e-07^{*}$ \\
\hline Q2 Expressed human transcripts first $150 \mathrm{bp}$ & 1.0 & $6.4 \mathrm{e}-01$ & $2.8 \mathrm{e}-2$ & $3.3 e-07^{*}$ \\
\hline Q3 Expressed human transcripts first $150 \mathrm{bp}$ & 1.0 & $6.1 \mathrm{e}-01$ & $7.6 e-2$ & $1.0 \mathrm{e}-06^{*}$ \\
\hline Q4 Expressed human transcripts first $150 \mathrm{bp}$ & 1.0 & $2.4 \mathrm{e}-01$ & $4.6 e-2$ & $9.7 e-07^{*}$ \\
\hline
\end{tabular}

${ }^{*}=p<0.005 ;$ NA not applicable

expressed quartiles (Q3 \& Q4) (Table 2). In addition, with a less stringent but acceptable significance level of 0.05 , the \%GC for the lowest two quartiles (Q1 \& Q2) are likely significantly different than the human fragment consensus sequences (Table 2).

In addition to GC-content, the enrichment of guanine suggests that these are purine-rich sequences, which have been shown to have a role in regulating transcription [19-21]. Therefore, the percentage of bases that are purines (\%AG) for each fragment was calculated using the transcribed strand of each gene and compared to various references using the WRT (Table 2, Additional file 3: Figure S3). The median \%AG for the human and bacterial fragment consensus sequences was 55.8 \& 61.7, respectively. The \%AG of the bacterial fragment consensus sequences was significantly different than the Pseudomonas $16 \mathrm{~S} \& 23 \mathrm{~S}$ rRNA gene database, and both of the complete and first $150 \mathrm{bp}$ of the expressed human transcripts in the participants with the bacterial DNA integrations (Table 2, $p<0.005$, WRT). On the other hand, the \%AG of the human fragment consensus sequences were statistically different than the expressed transcripts from the participants with the bacterial DNA integrations (Table 2, $p<0.005$, WRT).
Due to the observed higher guanine content of these sequences, the human genes with bacterial DNA integrations were investigated for guanine associated motifs. A database search of published G-quartets in the human genome [22] did not reveal evidence for G-quartets near the integrations. Examination of the first exon and 1 kbp upstream of the TSS for CEACAM5, CEACAM6, and CD74 did not reveal CpG islands (Additional file 4: Figure S4A-F). On the other hand, all three algorithms support $\mathrm{CpG}$ islands in the $1 \mathrm{kbp}$ region upstream of the TMSB10 TSS. However, only 2 of the 3 algorithms suggested the $\mathrm{CpG}$ island may extend downstream of the TSS near the predicted integration into TMSB10 (Additional file 4: Figure S4G). CpG islands are typically defined as $\geq 200$ bp regions that have more observed than expected CpG dinucleotides (>0.6) and high GC content $(>0.5)$. Next, the window size for identifying $\mathrm{CpG}$ islands was decreased to refine the location of the CpG island signal in the first exon of TMSB10. The CpG signal was determined to originate from the 3 ' end of the first exon of TMSB10, while the side of the first exon near the predicted integration site had no CpG signal (Additional file 4: Figure S4H). Therefore, it is unlikely the bacterial integration into TMSB10 disrupted CpG sites. 


\section{rRNA structure}

Based on the position of the bacterial DNA integrations into $5^{\prime}$-UTR of the human genes, it is possible that the bacterial DNA integrations altered the expression of the human genes by disrupting a regulatory element in the $5^{\prime}$-UTR. One reason to expect that this might be the case is that the integrations arise from the $16 \mathrm{~S} \& 23 \mathrm{~S}$ rRNA genes that contain numerous stem-loop structures that have the potential to alter secondary structure and thus transcription. Therefore, the consensus sequence of the integrated bacterial DNA was examined for the presence of stem-loop structures from the bacterial rRNA gene by mapping the bacterial fragments onto the known secondary structure (Additional file 5: Figure S5). It was concluded that $11 / 14$ integrations have large stem-loop structures in the middle of the bacterial fragments; while the remaining three fragments of integrated bacterial DNA have stem-loop structures near the ends of each fragment.

Subsequently, the predicted RNA structure of the human transcripts was examined to determine if the bacterial integrations may have disrupted the secondary structure of the human transcripts. By mapping the predicted position of each integration onto the CEACAM5, CEACAM6, CD74, and TMSB10 predicted mRNA transcript structures, it was determined that the integrations are either in, or near, complex secondary structures (Additional file 6: Figure S6).

\section{Relative position of integration}

To explore if the bacterial DNA integrations may be occurring in the same relative position within each human gene, the relative position of the predicted site for integration within the first exon was calculated (Table 3). While the CEACAM5 and CEACAM6 integrations are enriched around the middle of the first exon, the two genes also share homology and have similar structures. The CEACAM genes, CD74, and TMSB10 all have different relative position of integrations (Table 3 ).

\section{Discussion}

\section{Obstacles in assembling the integrations}

Here, the Jensen-Shannon distance between the insertsize distributions of the paired-end reads supporting the Pseudomonas-like DNA integrations relative to the respective sequencing library was used to model bacterial DNA integration in CEACAM5, CEACAM6, CD74, \& TMSB10. The JSD values supporting these models are within the expected range of the JSD values given the number of reads supporting the bacterial DNA integrations.

Ideally, the sequencing reads would be assembled to determine the exact integration site and sequence. There are likely three compounding factors that prevent the
Table 3 Relative position of the bacterial DNA integrations

\begin{tabular}{lll}
\hline Name & $\begin{array}{l}\text { Absolute distance } \\
\text { of the integration } \\
\text { from TSS (bp) }\end{array}$ & $\begin{array}{l}\text { Relative distance of } \\
\text { the integration from } \\
\text { the TSS of } 1^{\text {st }} \text { Exon (\%) }\end{array}$ \\
\hline A_16S_C5 & 61 & 50 \\
A_23S1_C5 & 62 & 51 \\
A_23S2_C5 & 64 & 53 \\
B_16S_C5 & 18 & 15 \\
B_16Sa_C5 & 50 & 41 \\
B_23S_C5 & 76 & 62 \\
A_16S_C6 & 64 & 45 \\
C_16S_C6 & 62 & 41 \\
D_16S1_CD & 184 & 79 \\
D_16S2_CD & 160 & 69 \\
D_23S_CD & 182 & 78 \\
E_16S_CD & 185 & 80 \\
E_23S1_CD & 166 & 72 \\
E_23S2_CD & 181 & 78 \\
F_16S_T10 & 4 & 4 \\
\hline
\end{tabular}

assembly of these bacterial DNA integrations. First, the reads are only $51 \mathrm{bp}$. In order to find a read that is on the integration breakpoint, the read on the breakpoint needs to be able to be split into uniquely human and bacterial portions. As a result of the short read length, a read would have to be exactly half human and half bacterial in order to give a sufficient sequence length of $\sim 25$ bp to identify each portion. This is highly unlikely to occur. Second, there is limited coverage of the integrations. The limited coverage is likely due to the proximity of the integrations to the beginnings of transcripts where RNA-Seq coverage is poor. In addition, it is possible that the low RNA-Seq coverage is the result of the bacterial DNA integrations inducing decreased expression. Lastly, the data suggest a heterogeneous population of transcript variants in a tumor that makes de novo assembly difficult.

In a mock-experiment with an artificial bacterial-human DNA integration, LGTSeek identified only 3 (3\%) reads that cover the breakpoint. These reads align to the bacterial DNA, with only 1-3 bp at the 3'-end of the read covering the breakpoint. LGTSeek uses a version of BWA that may not identify these reads. While a more recent version of BWA identifies soft-clipped reads better, it also works on the assumption that all reads should match the reference and as such has been found to erroneously align bacterial reads to the human reference. In KPL1, the cloned and sequenced bacteria-human DNA junction was used to generate a similar mock dataset that demonstrates that split and soft-clipped reads could not be identified due to a 24-bp inversion. Given that when the bacterial and human DNA are flush, only $3 \%$ of read pairs are identified, 
and that there are only 5 read pairs supporting STAD bacterial DNA integrations on average, we do not expect that soft-clipped reads would be identified even if the bacterial DNA is flush to the human DNA. Furthermore, such soft clipped reads that do exist would be difficult to distinguish from sequencing error or sequencing polymorphism between the bacterial sequence and the reference genome. Additionally, the average distance between the bacterial and human fragments of DNA in the models described here was found to be $\sim 24 \mathrm{bp}$, which suggests that the two may not be flush, further explaining the difficulty in detecting such reads.

\section{Heterogeneous integrations}

Participants A through E all have data supporting multiple integrations of different fragments of the $16 \mathrm{~S}$ and 23S rRNA Pseudomonas gene into the same human gene within each participant. In addition, the data support multiple participants with similar, if not identical, bacterial DNA integrations. For example, participants A \& B have $16 \mathrm{~S}$ and $23 \mathrm{~S}$ rRNA integrations into the $50-70 \mathrm{bp}$ of CEACAM5. This latter result suggests that the tumors sequenced have a heterogeneous population of cancer cells with different integrations.

\section{Characterizing the integrations}

These bacterial DNA integrations were investigated to determine if they are enriched in absolute position as well as relative position within the UTR and first exon of each gene. However, no pattern for the position of integration into these genes was identified, suggesting that each gene may have a different feature that is affected by, or permissive to, the bacterial DNA integrations.

The bacterial and human fragments supporting the integrations are high in guanine, demonstrated by the combination of high \%GC and high \%AG. Despite the high guanine content, it does not appear that the bacterial DNA integrations disrupted $G$ quartets or $\mathrm{CpG}$ islands, but the disruption of other G-rich motifs cannot be ruled out.

\section{Functional consequences of the bacterial DNA integrations}

The integrations of the Pseudomonas 16S and 23S rRNA genes may be important and have a biological effect by altering the transcriptional regulation of the human genes. One possibility is that the integration of bacterial DNA introduced new structures at the breakpoint between bacterial and human DNA that alter the transcriptional regulation of these human genes. Given that all of the bacterial integrations have stem-loop structures, secondary structure may play an important role. Alternatively, the integrated bacterial sequence could alter the regulation of these genes by disrupting either the transcript stability, or the availability of the transcriptional and/or translation machinery to bind to these genes.

Considering that the integrations are into the 5'-UTR of these genes, before the translational start site, it is unlikely that the integrations introduced a frameshift or premature stop codon. It is also unlikely that these integrations have interfered with splicing since the integrations are not near a splice site recognition sequence. However, it cannot ruled out that the integrations have created novel transcriptional start sites, translational start sites, or splice sites that would have functional consequences.

\section{Conclusion}

Based on the available data, models were generated for the bacterial $16 \mathrm{~S}$ and $23 \mathrm{~S}$ rRNA fragment integrations into the human CEACAM5, CEACAM6, CD74, and TMSB10. These mutations are especially intriguing because of their positions in the $5^{\prime}$-UTR near the transcriptional start site. The models presented here lay the groundwork for further in vitro experiments reconstructing these sequences to test if the bacterial rRNA integrations may alter the expression of the human genes.

\section{Methods}

\section{Library insert-size}

The sequencing reads were aligned to the human RefSeq transcriptome reference (available for download 02/22/ 2013) [23] using BWA v.0.5.9-r16 [24] with the default settings. Picard [25] was used to calculate each participant's insert-size for the library with default settings. Only the forward-reverse mapped reads were used in the insert-size calculation. The median and absolute deviations of the insert-size were used because the distribution of the data was typically asymmetric. A consistent color scheme (Additional file 7: Figure S7) was used in the figures to illustrate how a given read pairs insert size relates to the distirbution of inserts sizes in the library.

\section{Average difference}

The consensus sequence for the bacterial and human fragments of DNA that support an integration was determined by mapping the appropriate reads to a reference using the farthest upstream and downstream reads to mark the boundaries of each fragment. The consensus sequence of each fragment was determined from the alignment using samtools and bcftools [26, 27]. All the coordinates reported here are based on the alignment of the reads to: Pseudomonas 16S rRNA [GenBank:M34133.1], Pseudomonas 23S rRNA [GenBank:Y00432.1], CEACAM5 [GenBank:NM_004363.3], CEACAM6 [GenBank:NM_002 483.6], CD74 [GenBank:NM_001025159.2], or TMSB10 [GenBank:NM_021103.3]. The appropriate bacterial and human fragments were then placed directly next to each 
other, the reads supporting the integration were aligned to these adjacent fragments, and the insert-sizes were calculated for each read pair. The AD was then calculated by averaging the difference between the insert-size of the read pairs, $I_{i}(x)$, and the median insert-size of the corresponding sequencing library, $M_{S L}$. This process was repeated recursively by adding 1 ambiguous base between the bacterial and human fragments. By adding 0-100 bp bases between the two fragments, the number of bases, $x$, between the two fragments was titrated to yield the minimum AD. The one exception for this process was for participant D, as the bacterial reads wouldn't align to these specific $16 \mathrm{~S}$ and $23 \mathrm{~S}$ rRNA references with the BWA default settings, despite OTUs parsimonious with a Pseudomonas-like bacterium. Instead, to maintain a consistent bacterial references, a default BLAST [28] search was performed to align the bacterial reads from participant $\mathrm{D}$ to these specific $16 \mathrm{~S}$ and $23 \mathrm{~S}$ rRNA references.

\section{Jensen-Shannon distance}

For the JSD, the number of bases, $x$, was titrated between the bacterial and human fragments to yield the minimum JSD [29] for the insert-size distributions of the integration model $\left(d_{I}(x)\right)$ and the library $\left(d_{S L}\right)$ as implemented by Arumugam et al. [30, 31]. Briefly, a probability distribution was calculated for the insert-sizes (the number of reads with a given insert-size/total number of reads) of the model and reference data. A pseudocount of 0.0000001 was used to replace any insert-sizes with zero reads to avoid having zero values in either the nominator or denominator. Using these two distributions, the JSD was calculated between the model and library insert-size distributions. Bootstrap support for the model was calculated using 1000 iterations of calculating the confidence interval of the JSD. The JSD calculations were made with a custom Perl [32] script (Additional file 8: Text S1) that used the Statistics::R module [33], R [34], Bio::Perl [35], and samtools [26, 27]. To calibrate the JSD, paired-end reads from participant B's library were randomly sampled using the Picard [25] DownsampleSam function.

\section{Cancer cell line analysis}

Using the LGTSeek pipeline [13], two cancer cell line datasets were analyzed for evidence of bacterial DNA integrations into the human genome. One dataset (EGAD00001000725) was generated by Genentech, Inc and Genentech Research and Early Development [17] and is made available through the European GenomePhenome Archive [36]. The other dataset was the Cancer Cell Line Encyclopedia [16] that was generated by a collaboration between the Broad Institute and Novartis Institutes for Biomedical Research and its Genomics Institute of the Novartis Research Foundation. The Cancer
Cell Line Encyclopedia is publicly available through the University of California Santa Cruz Genomics Institute Cancer Genomics Hub [37].

\section{Validating the KPL-1 integration}

The KPL-1 integration junction was PCR amplified with primers 5'-GGCTACCCGTGATATTGCTG-3' and 5' AGGTTTCAGCTGGTTTTTGC-3' targeting the consensus bacterial and human fragments, respectively. The PCR was performed using Taq 2x Master Mix (New England BioLab, Ipswich, MA, USA) with $0.2 \mu \mathrm{M}$ primer (Sigma-Aldrich, St. Louis, MO, USA) and 20 ng of KPL-1 genomic DNA (DSMZ, Braunschweig, Germany). Template was denatured at $95^{\circ} \mathrm{C}$ for $60 \mathrm{~s}$ followed by 30 cycles of denaturing at $95^{\circ} \mathrm{C}$ for $20 \mathrm{~s}$, annealing at $60^{\circ} \mathrm{C}$ for $30 \mathrm{~s}$, and elongation at $68^{\circ} \mathrm{C}$ for $40 \mathrm{~s}$, followed by a final $5 \mathrm{~min}$ elongation at $68^{\circ} \mathrm{C}$. The resulting PCR product was then directly cloned into $\mathrm{pCR}$ 2.1-TOPO vector using the TOPO TA cloning kit following the manufacturer's protocol followed by transformation into One Shot TOP10 chemically competent cells (Life Technologies, Grand Island, NY, USA). Plasmid was isolated from an overnight culture in $\mathrm{LB}$ with $0.1 \mu \mathrm{g} / \mu \mathrm{L}$ carbenicillin from a single isolated colony with the QIAprep Spin Miniprep kit (Qiagen, Valencia, CA, USA) following the manufacturer's protocol. The plasmid was sequenced using standard M13 forward and reverse primers at the University of Maryland Institute for Genome Science Genome Resource Center and analyzed using CLC Genomics Workbench v.7.

\section{Guanine calculations}

The \%GC and \%AG were calculated using custom perl scripts to the complete bacterial references for the genus Pseudomonas obtained from RefSeq (Additional file 9: Text S2), the $16 \mathrm{~S}$ and $23 \mathrm{~S}$ rRNA gene references for the genus Pseudomonas from the Silva database release 119 (http://www.arb-silva.de/), the hg38 human genome reference, and the hg38 transcriptome reference. The RPKM was calculated using custom perl scripts for the participants with bacterial DNA integrations. Transcripts with $>0$ RPKM were considered expressed. Differences between the $\% \mathrm{GC}$ or \%AG of the bacterial or human fragment consensus sequences and the references were tested using the Wilcoxon rank-sum test and Bonferroni corrected. The data are illustrated in Additional file 2: Figure S2 using R \& the ggplot 2 boxplot function. The outliers were not removed from any calculations. The expression data were stratified so that the lowest expressed transcripts are in Q1, while the highest expressed transcripts are in Q4.

\section{CpG islands and G-quartets}

The non-B database [22] was searched for G-quartets in CEACAM5, CEACAM6, CD74, and TMSB10. The database did not report any identified motifs in the first exon 
of these genes using the search criteria "G Quadruplex Motif."

The human genes with integrations were searched for CpG islands using: CpGProD [38], EMBOSS [39-41], and SMS [42]. Given that CpG islands are typically defined as $\geq 200 \mathrm{bp}$, the first exon of each gene and $1 \mathrm{kbp}$ upstream of the TSS were searched for predicted CpG islands. The following references and coordinates for each gene were used for the CpG island searches: $C E A$ CAM5 [GenBank:NC_000019.10, 41707611-41708795], CEACAM6 [GenBank:NC_000019.10, 41754490-41755703], CD74 [GenBank:NC_000005.10, 150412625-150413936], and TMSB10 [GenBank:NC_000002.12, 84904639-84905718]. In order to analyze only the first exon for CpG islands, the EMBOSS algorithm settings were set so that the window size was $10 \mathrm{bp}$ (default $100 \mathrm{bp}$ ) and a CpG island minimum length was $20 \mathrm{bp}$. The first exons were defined as: $C E A$ CAM5 [GenBank:NC_000019.10, 41708611-41708795], CEACAM6 [GenBank:NC_000019.10, 41755490-41755703], CD74 [GenBank:NC_000005.10, 150412625-150412936], and TMSB10 [GenBank:NC_000002.12, 84905639-84905718].

The average \%GC content of the Pseudomonas genomes was calculated on all RefSeq Pseudomonas complete genomes available from RefSeq (release 01.05.2015). The \%GC for the Pseudomonas $16 \mathrm{~S}$ and 23S rRNA genes was calculated using all Pseudomonas rRNA genes from the SILVA rRNA database (release 119) [43]. The average \%GC content of the human genome was calculated on all the nuclear chromosomes using hg19 and the RefSeq transcriptome (downloaded 04.01.15).

\section{Secondary structure}

The Pseudomonas 16S [GenBank: M34133.1] and 23S [GenBank: Y00432.1] rRNA secondary structures were downloaded from the Comparative RNA Web Site and Project [44]. The human secondary structures of $C E A$ CAM5, CEACAM6, CD74, and TMSB10 were predicted using the minimum free energy prediction by the RNAfold server $[45,46]$.

\section{Relative position of integrations}

The absolute distance between the integration site and the TSS was calculated by taking the difference (bp) between the middle of each predicted site of integration and the TSS for the respective gene (Table 2, column 2). To calculate the relative position, the difference was divided by the total size of the first exon (bp) and multiplied by 100 to get the percent distance the integration is relative to the TSS and the end of the first exon (Table 2, column 3).

\section{Availability of data and materials}

The datasets supporting the conclusions of this article are available in The Cancer Genome Atlas (TCGA) [phs000178, https://cghub.ucsc.edu] (Additional File
10: Table S1), the Cancer Cell Line Encyclopedia (CCLE) [16] [CCLE, https://cghub.ucsc.edu], and Genentech Inc [17] [EGAD00001000725, https://www.ebi.ac.uk/ega/home]. Information about TCGA, the investigators, and the institutions who constitute the TCGA research network can be found at "http://cancergenome.nih.gov". The TCGA data were obtained via the Sequence Read Archive as approved by dbGap. The CCLE data were generated by the Broad Institute and Novartis Institutes for Biomedical Research and its Genomics Institute of the Novartis Research Foundation Data. The CCLE data were made publicly available through the University of California Santa Cruz Genomics Institute CGHub. Information about the CCLE can be found at "https://cghub.ucsc. edu/datasets/ccle.html". The EGAD00001000725 data were generated by Genentech Inc and Genentech Research and Early Development. The University of Maryland, Baltimore, Institutional Review Board reviewed this study and determined that it did not require IRB review.

\section{Additional files}

\begin{abstract}
Additional file 1: Figure S1. Confirming the CEACAM5 transcriptional start site annotation. The expression level around the TSS of CEACAM5 is compared across all previously analyzed participants in the stomach adenocarcinoma and breast cancer data sets to that of the well-characterized ACTB. The CEACAM5 expression is consistent with an accurate TSS annotation in the human genome. (PDF $3219 \mathrm{~kb}$ )
\end{abstract}

Additional file 2: Figure S2. KPL1 breakpoint. An illustration of the junction of bacterial and human DNA from the KPL1 cell line. The breakpoint consists of bacterial DNA with homology to an aminoglycoside phosphotransferase gene, such as in the Escherichia coli K12. The human DNA is homologous to chromosome 6 . There is a 24 bp bacterial inversion with overlap at the breakpoint. The bacterial and human DNA are $99 \%$ (194/195 \& 24/24) and $100 \%$ (200/200) identical, respectively. (PDF 823 kb)

Additional file 3: Figure S3. Guanine enrichment near bacterial DNA integrations. The distributions of the \%GC and \%AG (panels A \& B, respectively) of the bacterial and human fragment consensus sequences were compared to other bacterial and human references. The expressed transcripts (>0 RPKM) in the participants with the bacterial DNA integrations were stratified based on RPKM into four quartiles $(\mathrm{Q} 1=$ red, $\mathrm{Q} 2=$ yellow, Q3 = green, Q4 = blue), with the lowest expressed transcripts in the first quartile (Q1) and the highest expressed transcripts in the fourth quartile (Q4). (PDF $158 \mathrm{~kb}$ )

Additional file 4: Figure S4. EMBOSS CpG island search results. Using the EMBOSS CpG island prediction software, the CEACAM5, CEACAM6, CD74, \& TMSB10 bacterial DNA integrations were not found to be in proximity to CpG islands (A-F). The default EMBOSS algorithm was used to search the first exon and $1 \mathrm{kbp}$ upstream of the TSS for each gene $(A, C, E, \& G)$, while altered parameters were used to search the first exon only (B,D,F,\&H). (PDF 539 kb)

Additional file 5: Figure S5. RNA secondary structure of the bacterial $16 S$ \& 235 integrated rRNA gene fragments. Each colored line indicates a fragment of the Pseudomonas 165 or $23 \mathrm{~S}$ rRNA gene that is predicted to have integrated into the human genome. The lines are color-coded based on the participant and human gene the bacterial rRNA fragment that has integrated into ( $C 5=$ CEACAM5, C6 = CEACAM6, CD = CD74, T10 = TMSB10, "_1" are upstream rRNA fragments relative to the "_2" integrations). (PDF $2295 \mathrm{~kb}$ ) 
Additional file 6: Figure S6. The predicted secondary structure of the human transcripts near the bacterial DNA integrations. The secondary structure for the examined human transcripts was predicted with the minimum free energy prediction from the RNAfold server (ViennaRNA v.2.2.0c) [45], and the location of the predicted integration of the Pseudomonas rRNA gene has been highlighted. (PDF $4110 \mathrm{~kb}$ )

Additional file 7: Figure S7. Schematic of the paired-end reads color scheme. An illustration of the color scheme used to describe the relationship between the insert-size for the paired-end reads (i) and the median insert-size of a participant's library. Lighter colors indicate the read pair's insert-size is closer to the median library insert-size. For the purpose of this illustration, these hypothetical reads have a median insert-size of $200 \mathrm{bp}$ and median absolute deviation ( $\sigma)$ of 20 bp. (PDF $171 \mathrm{~kb}$ )

Additional file 8: Text S1. Code to calculate the models of DNA integration, AD \& JSD. This code is a part of a custom Perl script that creates the models for an integration. Specifically, this code calculates the consensus sequence and the optimum distance between the two fragments using the average difference and Jensen-Shannon Distance. (PDF $53 \mathrm{~kb}$ )

Additional file 9: Text S2. Pseudomonas accessions. A list of GenBank accessions used to calculate the Pseudomonas genome \%GC. (TXT 672 bytes)

Additional file 10: Table S1 Conversion of the participant names to SRR accession number. (DOC $66 \mathrm{~kb}$ )

\section{Abbreviations}

AD: Average difference; BRCA: Breast cancer; CCLE: Cancer Cell Line Encyclopedia; JSD: Jensen-Shannon distance; STAD: Stomach adenocarcinoma; TCGA: The Cancer Genome Atlas; TSS: Transcriptional start site.

\section{Competing interests}

The authors declare that they have no competing interests.

\section{Author contributions}

KBS designed the study, conducted the bioinformatics analysis, prepared figures, analyzed the data, and wrote the manuscript. PG participated in study design, data analysis, figure preparation, and editing the manuscript. JCDH was responsible for study design, data analysis, and editing the manuscript. All authors read and approved the final manuscript.

\section{Acknowledgements}

This work was funded by the National Institutes of Health through the NIH Director's New Innovator Award Program (1-DP2-OD007372), NIH Director's Transformative Research Award (1-R01-CA206188), and NIDDK Gl-T32 DK067872-10

\section{Author details}

${ }^{1}$ Institute for Genome Science, University of Maryland School of Medicine, Baltimore, MD 21201, USA. Department of Microbiology and Immunology, University of Maryland School of Medicine, Baltimore, MD 21201, USA. ${ }^{3}$ Greenebaum Cancer Center, University of Maryland School of Medicine, Baltimore, MD 21201, USA

\section{Received: 25 August 2015 Accepted: 9 March 2016}

Published online: 21 March 2016

\section{References}

1. Huang FW, Hodis E, Xu MJ, Kryukov GV, Chin L, Garraway LA. Highly recurrent TERT promoter mutations in human melanoma. Science. 2013;339(6122):957-9.

2. Horn S, Figl A, Rachakonda PS, Fischer C, Sucker A, Gast A, Kadel S, Moll I,

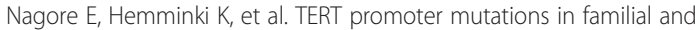
sporadic melanoma. Science. 2013;339(6122):959-61.

3. Banerji S, Cibulskis K, Rangel-Escareno C, Brown KK, Carter SL, Frederick AM, Lawrence MS, Sivachenko AY, Sougnez C, Zou L,et al. Sequence analysis of mutations and translocations across breast cancer subtypes. Nature. 2012:486(7403):405-9.

4. Newburger DE, Kashef-Haghighi D, Weng Z, Salari R, Sweeney RT, Brunner AL, Zhu SX, Guo X, Varma S, Troxell ML, et al. Genome evolution during progression to breast cancer. Genome Res. 2013;23(7):1097-108.
5. Malhotra A, Lindberg M, Faust GG, Leibowitz ML, Clark RA, Layer RM, Quinlan AR, Hall IM. Breakpoint profiling of 64 cancer genomes reveals numerous complex rearrangements spawned by homology-independent mechanisms. Genome Res. 2013;23(5):762-76.

6. Korbel JO, Urban AE, Affourtit JP, Godwin B, Grubert F, Simons JF, Kim PM Palejev D, Carriero NJ, Du L, et al. Paired-end mapping reveals extensive structural variation in the human genome. Science. 2007;318(5849):420-6.

7. Welch JS, Westervelt P, Ding L, Larson DE, KIco JM, Kulkarni S, Wallis J, Chen K, Payton JE, Fulton RS, et al. Use of whole-genome sequencing to diagnose a cryptic fusion oncogene. JAMA. 2011;305(15):1577-84.

8. Sung WK, Zheng H, Li S, Chen R, Liu X, Li Y, Lee NP, Lee WH, Ariyaratne PN, Tennakoon C, et al. Genome-wide survey of recurrent HBV integration in hepatocellular carcinoma. Nat Genet. 2012;44(7):765-9.

9. Landry JJ, Pyl PT, Rausch T, Zichner T, Tekkedil MM, Stutz AM, Jauch A, Aiyar RS, Pau G, Delhomme N, et al. The genomic and transcriptomic landscape of a HeLa cell line. G3 (Bethesda). 2013;3(8):1213-24.

10. Tubio JM, Li Y, Ju YS, Martincorena I, Cooke SL, Tojo M, Gundem G, Pipinikas CP, Zamora J, Raine K, et al. Mobile DNA in cancer. Extensive transduction of nonrepetitive DNA mediated by L1 retrotransposition in cancer genomes. Science. 2014;345(6196):1251343.

11. Baillie JK, Barnett MW, Upton KR, Gerhardt DJ, Richmond TA, De Sapio F, Brennan PM, Rizzu P, Smith S, Fell M, et al. Somatic retrotransposition alters the genetic landscape of the human brain. Nature. 2011;479(7374): 534-7.

12. Ewing $A D$, Kazazian Jr HH. High-throughput sequencing reveals extensive variation in human-specific L1 content in individual human genomes. Genome Res. 2010;20(9):1262-70.

13. Riley DR, Sieber KB, Robinson KM, White JR, Ganesan A, Nourbakhsh S, Dunning Hotopp JC. Bacteria-human somatic cell lateral gene transfer is enriched in cancer samples. PLoS Comput Biol. 2013;9(6):e1003107.

14. Kloosterman WP, Tavakoli-Yaraki M, van Roosmalen MJ, van Binsbergen E, Renkens I, Duran K, Ballarati L, Vergult S, Giardino D, Hansson K, et al. Constitutional chromothripsis rearrangements involve clustered doublestranded DNA breaks and nonhomologous repair mechanisms. Cell Rep. 2012;1(6):648-55.

15. Drier Y, Lawrence MS, Carter SL, Stewart C, Gabriel SB, Lander ES, Meyerson M, Beroukhim R, Getz G. Somatic rearrangements across cancer reveal classes of samples with distinct patterns of DNA breakage and rearrangement-induced hypermutability. Genome Res. 2013;23(2):228-35.

16. Barretina J, Caponigro G, Stransky N, Venkatesan K, Margolin AA, Kim S, Wilson CJ, Lehar J, Kryukov GV, Sonkin D, et al. The cancer cell line encyclopedia enables predictive modelling of anticancer drug sensitivity. Nature. 2012:483(7391):603-7.

17. Klijn C, Durinck S, Stawiski EW, Haverty PM, Jiang Z, Liu H, Degenhardt J, Mayba O, Gnad F, Liu J, et al. A comprehensive transcriptional portrait of human cancer cell lines. Nat Biotechnol. 2015;33(3):306-12.

18. Kudla G, Lipinski L, Caffin F, Helwak A, Zylicz M. High guanine and cytosine content increases mRNA levels in mammalian cells. PLoS Biol. 2006;4(6):e180.

19. Kennedy GC, Rutter WJ. Pur-1, a zinc-finger protein that binds to purine-rich sequences, transactivates an insulin promoter in heterologous cells. Proc Natl Acad Sci U S A. 1992;89(23):11498-502.

20. Gupta M, Zak R, Libermann TA, Gupta MP. Tissue-restricted expression of the cardiac alpha-myosin heavy chain gene is controlled by a downstream repressor element containing a palindrome of two ets-binding sites. Mol Cell Biol. 1998;18(12):7243-58.

21. Rumora AE, Wang SX, Ferris LA, Everse SJ, Kelm Jr RJ. Structural basis of multisite single-stranded DNA recognition and ACTA2 repression by purine-rich element binding protein B (Purbeta). Biochemistry. 2013; 52(26):4439-50

22. Cer RZ, Donohue DE, Mudunuri US, Temiz NA, Loss MA, Starner NJ, Halusa GN, Volfovsky N, Yi M, Luke BT, et al. Non-B DB v2.0: a database of predicted non-B DNA-forming motifs and its associated tools. Nucleic Acids Res. 2013; 41:D94-D100.

23. Pruitt K, Brown G, Tatusova T, Maglott D. The Reference Sequence (RefSeq) Project. In: M J, O J, editors. The NCBI handbook [Internet]. Bethesda MD: National Library of Medicine (US), National Center for Biotechnology Information; 2002. http://www.ncbi.nlm.nih.gov/books/NBK21091/.

24. Li H, Durbin R. Fast and accurate short read alignment with Burrows-Wheeler transform. Bioinformatics. 2009:25(14):1754-60.

25. Picard. 1.124 edn. http://broadinstitute.github.io/picard/. 
26. Li H, Handsaker B, Wysoker A, Fennell T, Ruan J, Homer N, Marth G, Abecasis G, Durbin R, Genome Project Data Processing S. The Sequence Alignment/ Map format and SAMtools. Bioinformatics. 2009;25(16):2078-9.

27. Li H. A statistical framework for SNP calling, mutation discovery, association mapping and population genetical parameter estimation from sequencing data. Bioinformatics. 2011;27(21):2987-93.

28. Altschul SF, Gish W, Miller W, Myers EW, Lipman DJ. Basic local alignment search tool. J Mol Biol. 1990;215(3):403-10.

29. Endres DM, Schindelin JE. A new metric for probability distributions. IEEE Trans Inform Theory. 2003:49(7):1858-60.

30. Arumugam M, Raes J, Pelletier E, Le Paslier D, Yamada T, Mende DR, Fernandes GR, Tap J, Bruls T, Batto JM, et al. Enterotypes of the human gut microbiome. Nature. 2011;473(7346):174-80.

31. Tutorial Enterotyping: the original publication. [http://enterotype.embl.de/ enterotypes.html]

32. Wall L. Perl. 5.8 .8 edn. http://www.perl.org

33. P GM. Statistics::R. 0.32 edn; 2014. http://search.cpan.org/ gmpassos/ Statistics-R-0.02/

34. Team RC. R: A Language and Environment for Statistical Computing. 310th ed. Vienna: R Foundation for Statistical Computing; 2014.

35. Stajich JE, Block D, Boulez K, Brenner SE, Chervitz SA, Dagdigian C, Fuellen G, Gilbert JG, Korf I, Lapp H, Lehväslaiho H, Matsalla C, Mungall CJ, Osborne Bl, Pocock MR, Schattner P, Senger M, Stein LD, Stupka E, Wilkinson MD, Birney E. The Bioperl toolkit: Perl modules for the life sciences. Genome Res. 2002;12(10):1611-8.

36. Lappalainen I, Almeida-King J, Kumanduri V, Senf A, Spalding JD, Ur-Rehman S, Saunders G, Kandasamy J, Caccamo M, Leinonen R, et al. The European Genome-phenome Archive of human data consented for biomedical research. Nat Genet. 2015;47(7):692-5.

37. Wilks C, Cline MS, Weiler E, Diehkans M, Craft B, Martin C, Murphy D, Pierce H, Black J, Nelson D, et al. The Cancer Genomics Hub (CGHub): overcoming cancer through the power of torrential data. Database. 2014;2014.

38. Ponger L, Mouchiroud D. CpGProD: identifying CpG islands associated with transcription start sites in large genomic mammalian sequences. Bioinformatics. 2002;18:631-3.

39. Rice P, Longden I, Bleasby A. EMBOSS: the European Molecular Biology Open Software Suite. Trends Genet. 2000;16(6):276-7.

40. Goujon M, McWilliam H, Li W, Valentin F, Squizzato S, Paern J, Lopez R. A new bioinformatics analysis tools framework at EMBL-EBI. Nucleic Acids Res. 2010;38(Web Server issue):W695-699.

41. McWilliam H, Li W, Uludag M, Squizzato S, Park YM, Buso N, Cowley AP, Lopez R. Analysis Tool Web Services from the EMBL-EBI. Nucleic Acids Res. 2013;41:W597-600.

42. Stothard P. The sequence manipulation suite: JavaScript programs for analyzing and formatting protein and DNA sequences. Biotechniques. 2000; 28:1102-4.

43. Quast C, Pruesse E, Yilmaz P, Gerken J, Schweer T, Yarza P, Peplies J, Glockner FO. The SILVA ribosomal RNA gene database project: improved data processing and web-based tools. Nucleic Acids Res. 2013:41(Database issue):D590-596.

44. Cannone JJ, Subramanian S, Schnare MN, Collett JR, D'Souza LM, Du Y, Feng B, Lin N, Madabusi LV, Muller KM, et al. The comparative RNA web (CRW) site: an online database of comparative sequence and structure information for ribosomal, intron, and other RNAs. BMC Bioinformatics. 2002;3:2.

45. Lorenz R, Bernhart SH, Honer Zu Siederdissen C, Tafer H, Flamm C, Stadler PF, Hofacker IL: ViennaRNA Package 2.0. Algorithms Mol Biol. 2011;6:26.

46. Hofacker IL, Fontana W, Stadler PF, Bonhoeffer LS, Tacker M, Schuster P. Fast folding and comparison of RNA secondary structures. Monatshefte für Chemie/Chemical Monthly. 1994;125(2):167-88.

\section{Submit your next manuscript to BioMed Central and we will help you at every step:}

- We accept pre-submission inquiries

- Our selector tool helps you to find the most relevant journal

- We provide round the clock customer support

- Convenient online submission

- Thorough peer review

- Inclusion in PubMed and all major indexing services

- Maximum visibility for your research

Submit your manuscript at www.biomedcentral.com/submit
C Biomed Central 